\title{
Surface hypothermia predicts murine mortality in the intragastric Vibrio vulnificus infection model
}

\author{
Hannah E. Gavin and Karla J. F. Satchell
}

\begin{abstract}
Background: The Gram-negative bacterium Vibrio vulnificus can cause severe disease in humans who consume undercooked, contaminated seafood. To study food-borne $V$. vulnificus disease in the laboratory, mouse virulence studies predominantly use death as the primary experimental endpoint because behaviorally based moribund status does not consistently predict lethality. This study assessed ventral surface temperature (VST) and its association with mouse survival during $V$. vulnificus virulence studies as an efficacious, humane alternative.

Methods: VST of mice intragastrically inoculated with $V$. vulnificus was measured every $2-\mathrm{h}$ for $24 \mathrm{~h}$ and data for minimal VST analyzed for prediction of lethal outcome.

Results: In contrast to the relatively stable VST of mock-infected control animals, mice infected with V. vulnificus exhibited hypothermia with minima occurring 8 to $12 \mathrm{~h}$ post-inoculation. The minimum VST of mice that proceeded to death was significantly lower than that of surviving mice. VST $\leq 23.5^{\circ} \mathrm{C}$ was predictive of subsequent death with a sensitivity of $68 \%$ and specificity of $95 \%$.

Conclusions: Use of VST $\leq 23.5^{\circ} \mathrm{C}$ as an experimental endpoint during V. vulnificus infection has potential to reduce suffering of nearly $70 \%$ of mice for a mean of $10 \mathrm{~h}$ per mouse, without compromising experimental efficacy. Temperature cutoff of $23.5^{\circ} \mathrm{C}$ exhibited $93 \%$ positive and $77 \%$ negative predictive value. For future $\mathrm{V}$. vulnificus virulence studies requiring only binary comparison (e.g., LD $_{50}$ assays), we find that VST can be applied as a humane endpoint. However, use of VST is not recommended when detailed survival kinetics are desired.
\end{abstract}

Keywords: Vibrio vulnificus, Enteric pathogen, Humane endpoint, Surface temperature, Infrared thermometry

\section{Background}

Vibrio vulnificus is a Gram-negative bacterium found in aquatic environments. It has the potential to cause disease in humans who encounter the bacterium $[1,2]$. Wound infections occur when open skin lesions are exposed to bacteria, usually during wading or swimming [3]. Gastrointestinal infections arise when humans consume raw or undercooked seafood - most frequently, shellfish - that contains the microbe [4]. In severe situations, these infections progress to necrotizing fasciitis and primary sepsis, respectively [5]. Survival outcomes are particularly poor for gastrointestinal $V$. vulnificus infections with mortality rates exceeding 50\%

\footnotetext{
* Correspondence: k-satchell@northwestern.edu

Department of Microbiology-Immunology, Northwestern University Feinberg School of Medicine, 303 E. Chicago Ave, Ward 6-205, Chicago, IL 60611, USA
}

of infected individuals [6]. In light of this infection severity, improved understanding of $V$. vulnificus pathogenesis is increasingly critical.

Controlled human case studies cannot be conducted due to low $V$. vulnificus treatment efficacy, and retrospective clinical analyses lack much of the detailed information necessary to obtain full comprehension of disease course [7-11]. Therefore, to study $V$. vulnificus foodborne infection, a mouse model is routinely employed [12, 13]. Following intragastric inoculation with a bacterial suspension, infected animals are monitored, usually over the course of 48-60 h, by which point they either succumb to or resolve the infection. In lethal cases, bacteria can be isolated from distal organs pre- and post-mortem, indicating this model recapitulates the bacterial dissemination observed in septic human disease [12, 14, 15]. 
While laboratory mouse experiments provide invaluable data on infectious diseases, animal distress is, in many cases, an inherent component of animal experimentation. For this reason, scientists as well as regulatory bodies have sought methods to limit suffering of murine research subjects [16]. Current data indicate that hypothermia is efficacious for predicting death due to late-stage infectious disease [17-20]. In addition, contactfree, infrared surface thermometers - as an alternative to rectal probe or implanted telemetric thermometers - are ideal for use in studies of acute gastrointestinal pathogenesis. Yet, notably, the specific temperature boundaries that predict infection lethality, and the utility of these boundaries, depend heavily upon the model system in question $[17,21]$. Therefore, this study was conducted to test the efficacy of hypothermia as a predictor of $V$. vulnificus mortality after oro-gastric inoculation, and to define the specific temperature parameters for implementation of hypothermia as a humane endpoint in $V$. vulnificus intragastric virulence studies.

\section{Methods}

\section{Bacterial strain selection}

These experiments were conducted using four strains of $V$. vulnificus that differ in the gene $r t x A 1$, which encodes the primary virulence toxin, the Multifunctional Autoprocessing Repeats-in-Toxins (MARTX) toxin. All strains are detailed in Table 1 and the toxin variants depicted schematically in Fig. 1a.

V. vulnificus strain HG0902 (CMCP6rif SvvhA::bla) was generated from the Korean clinical isolate $V$. vulnificus CMCP6rif. Although originally described as a genetic deletion of $v v h B A$ [12], subsequent gene mapping revealed disruption of only $v v h A$ by the stable integration of a portion of ampicillin-resistant plasmid pHGJ4. This strain was then further modified. Plasmids for alteration of $r t x A 1$ to $r t x A 1:: b l a, m c f:: b l a$, or $\Delta m c f$ have been described previously $[15,22,23]$. Each plasmid was

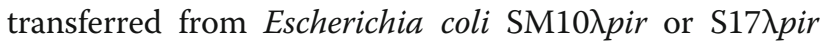
to $V$. vulnificus HG0902 by conjugation. Selection for double homologous recombination was conducted using sucrose counterselection to isolate recombinants as previously described [24]. Genetic modification was confirmed by polymerase chain reaction (PCR).

Table 1 Strains used in this study

\begin{tabular}{llll}
\hline & Name & Description & Reference \\
\hline 1 & HG0902 & CMCP6rif $\Omega v v h A:$ :bla & {$[12]$} \\
2 & HG1102 & HG0902 with rtxA1::bla & This study \\
3 & SNG1301 & HG9092 with mcf deletion in rtxA1 & This study \\
4 & SNG1302 & HG1102 with mcf reintroduced & This study \\
\hline
\end{tabular}

\section{Bacterial growth and preparation}

Bacteria were routinely grown in Luria-Burtani (LB) broth (10 g tryptone, 5 g yeast extract, $5 \mathrm{~g} \mathrm{NaCl}$ ) containing $50 \mu \mathrm{g} / \mathrm{mL}$ rifampin as needed. For all experiments, $V$. vulnificus was streaked from frozen glycerol stocks onto LBrif plates 2 days prior to experimentation. The following day, single colonies were picked and grown in $2 \mathrm{~mL} \mathrm{LB}$ broth with selective antibiotic overnight at $30{ }^{\circ} \mathrm{C}$ with shaking at $225 \mathrm{rpm}$. The following morning, strains were subcultured 1:100 into LB without antibiotic and grown to mid-log phase at $30{ }^{\circ} \mathrm{C}$ with shaking at $225 \mathrm{rpm}$. Cultures were pelleted and resuspended in sterile phosphate buffered saline (PBS, $10 \mathrm{mM}$ sodium phosphate, $140 \mathrm{mM} \mathrm{NaCl}, \mathrm{pH}$ 7.4) to indicated concentrations based on optical density $\left(\mathrm{A}_{600}\right)$. Bacterial inoculum concentration was confirmed by dilution plating.

\section{Mouse infection}

Fifty female C57BL/6 mice were obtained from Jackson Laboratories (Bar Harbor, ME). Animals were housed 5 per cage in wood shavings with disposable paper huts and cotton bedding squares as nesting material. Water and food were provided ad libitum. Experiments were conducted when mice were 5-6 weeks of age. For each of the two independent experiments, 25 mice were divided into 5 groups of 5 mice each to be either mockinoculated with PBS, or inoculated with one of the four $V$. vulnificus strains.

Each mouse was transiently anaesthetized using isoflurane and then inoculated intragastrically (i.g.) with $50 \mu \mathrm{L}$ of PBS or $1 \times 10^{8}$ colony-forming units (CFU) $V$. vulnificus suspended in PBS using a $1-\mathrm{cm}$ animal feeding needle attached to a 1-mL syringe. Mice were then injected intraperitoneally with $100 \mu \mathrm{L}$ of a cocktail containing $10 \mu \mathrm{g} / \mathrm{ml}$ ketamine and $2 \mu \mathrm{g} / \mathrm{ml}$ xylazine in PBS to facilitate bacterial infection [25].

Mice were monitored hourly for the first $24 \mathrm{~h}(\mathrm{~h})$ and subsequently every 4-8 h until 48 h post-infection (hpi). Ventral surface temperature (VST) was measured every $2 \mathrm{~h}$ during the first $24 \mathrm{~h}$ then once at $40-42 \mathrm{~h}$ and once at 47-48 h. Temperature was measured using the noncontact infrared TW2 thermometer (ThermoWorks, Alpine, UT) as recommended by the manufacturer. Thermometer emissivity maintained at the manufacturer's default setting of 0.95 . The TW2 thermometer is accurate $+/-1.0{ }^{\circ} \mathrm{C}$ when the object of interest is between 15 and $35^{\circ} \mathrm{C}$.

To obtain temperature measurements, mice were restrained by scruffing. The thermometer was held approximately $6 \mathrm{~cm}$ from the mouse ventral side and the beam aimed below the base of the sternum, according to procedures that have previously described practices for obtaining consistent temperature readings [26, 27]. 


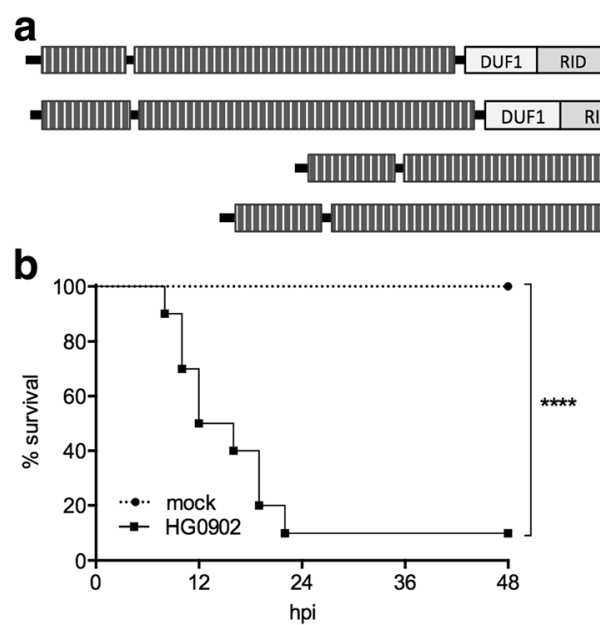

Fig. 1 a Schematic representation of the MARTX toxin variant produced by the $V$. vulnificus strain name indicated at right. b Survival of mice infected with HG0902 compared to mock-infected mice, $n=10$ /group. c Survival of mice infected with the indicated strains, $n=10 /$ group (note that mock and HG0902 curves are repeated from 1B). Survival curves were compared by log-rank test. ${ }^{* * *} p \leq 0.0001$

Because mouse temperature can change rapidly upon handling, efforts were made to minimize and standardize handling methods across all mice $[26,28,29]$. As such, a temperature stabilization time of $5-10 \mathrm{~s}$ was employed prior to temperature recording.

\section{Statistical analyses}

Statistical analyses were performed as indicated in figure legends using GraphPad Prism 6.0 software.

\section{Results}

\section{V. vulnificus strains show distinct survival patterns}

For pooled data across both experiments, all ten of the mock-infected animals survived to 48 hpi (Fig. 1b). In contrast, $90 \%$ of mice inoculated with the parental $\mathrm{V}$. vulnificus strain HG0902 succumbed to infection within 24 hpi (Fig. 1b), an outcome consistent with previous experiments $[12,15]$. In strain HG1102, the $r t x A 1$ gene is altered to replace MARTX toxin effector domains with a heterologous beta-lactamase coding region. All mice infected with HG1102 survived to 48 hpi demonstrating significant virulence attenuation (Fig. 1c). These results are consistent with previous data demonstrating that elimination of the MARTX effector domains attenuated virulence equivalent to an $r$ txA1-null mutant [15]. By contrast, deletion of only the $m c f$ coding region in SNG1301 did not significantly attenuate virulence compared to HG0902 (Fig. 1c). Similarly, the addition of $m c f$ in strain SNG1302 did not significantly enhance virulence of the attenuated strain HG1102 (Fig. 1c). Therefore, the MCF domain alone is neither necessary nor sufficient for MARTX toxinassociated virulence in $V$. vulnificus.
VST measurement profiles vary dependent upon survival outcome

Data were first analyzed pooling results for all $V$. vulnificus groups tested. Of 40 mice inoculated with any strain of $V$. vulnificus, 21 mice survived and 19 mice died. For non-survivors, the mean time-to-death (TTD) was 17 hpi (S.D. \pm 8 hpi) with a range of 8 to $40 \mathrm{hpi}$. In the course of these experiments, the VST was also measured (Fig. 2). Among all mice, the minimum VST $\left(\mathrm{VST}_{\text {min }}\right)$ reading during the experiment was $21.1{ }^{\circ} \mathrm{C}$ and the maximum VST (VST ${ }_{\text {max }}$ ) was $33.7^{\circ} \mathrm{C}$ for a total measurement range of $12.6{ }^{\circ} \mathrm{C}$.

During the first 0 to $4 \mathrm{hpi}$, the majority (46 of 50) of tested animals exhibited a drop in VST relative to initial $\left(\mathrm{VST}_{0}\right)$. This drop was measured in both infected and mock-treated animals (Fig. 2a-c, grey zones). The temperature decrease at this initial stage is attributed to anesthetic-induced hypothermia [30] as it occurred independent of infection status. For mock-treated animals, VST rebounded to match or exceed $\mathrm{VST}_{0}$, usually by 4 hpi and in all cases by 6 hpi (Fig. 2a). VST of mockinfected mice then generally remained stable after recovery from the initial drop (6-48 hpi).

Among $V$. vulnificus inoculated animals, measured VST of mice that survived spanned a wide range of more than $11{ }^{\circ} \mathrm{C}$ (Fig. 2b). Survivors most frequently exhibited $\mathrm{VST}_{\min }$ at 6 or $8 \mathrm{hpi}$. Subsequent VST readings in survivors were characterized by a period of temperature recovery $(8-14 \mathrm{hpi})$ and finally a period of temperature stability (16-48 hpi).

In contrast, measured VST of mice that succumbed to infection generally did not recover after the anesthetic period of 0-4 hpi (Fig. 2c). Instead, VST of nonsurvivors continued to decrease, spanned a much 


\section{a mock}

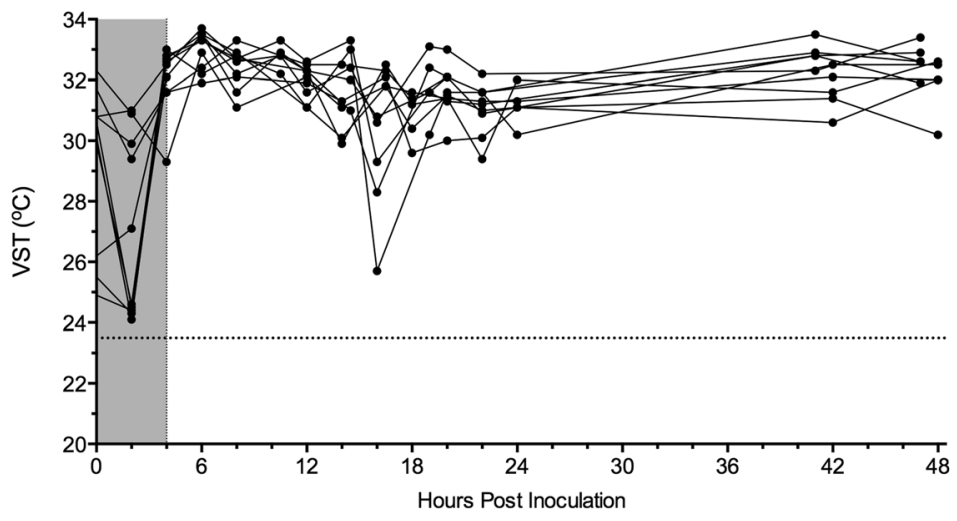

b survivors

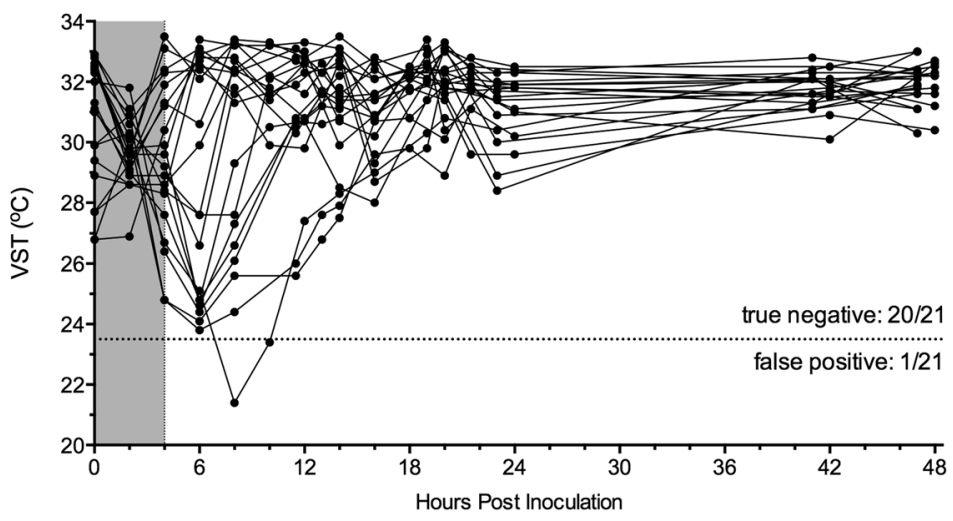

C non-survivors

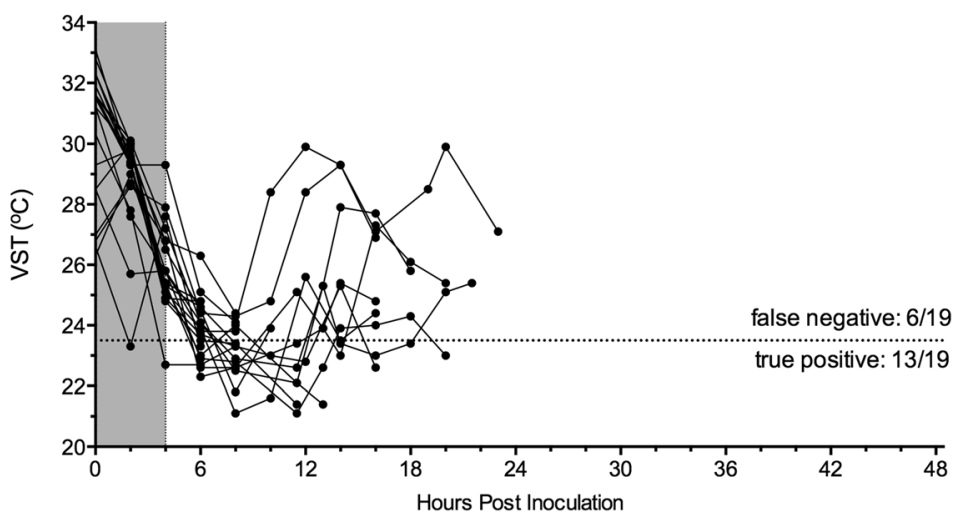

Fig. 2 Ventral surface temperature (VST) readings over time for: (a) mock infected mice, (b) mice that survived infection and (c) mice that did not survive infection. Each dot represents a single VST reading, and a continuous line connects VST readings from the same mouse. VST measurement ends either at 48 hpi or at time of death

smaller range of less than $4{ }^{\circ} \mathrm{C}$, and exhibited VST $\mathrm{T}_{\min }$ readings at $8-12$ hpi (Fig. 2c). In many cases, VST of non-survivors increased in the span between 12 and 18 hpi, despite the fact that these animals succumbed to infection by 24 hpi. This pattern is distinct both from the mock-treated group and infected animals that survive inoculation. Thus, VST measurement profiles vary depending upon both $V$. vulnificus infection status, and infection survival outcomes.

\section{VST of $23.5^{\circ} \mathrm{C}$ is associated with lethality during $V$.} vulnificus infection

Comparing VST between 6 and 48 hpi across groups, mean $\mathrm{VST}_{\text {min }}$ of surviving mice $\left(27.9 \pm 3.2{ }^{\circ} \mathrm{C}\right.$, Fig. 3$)$ 


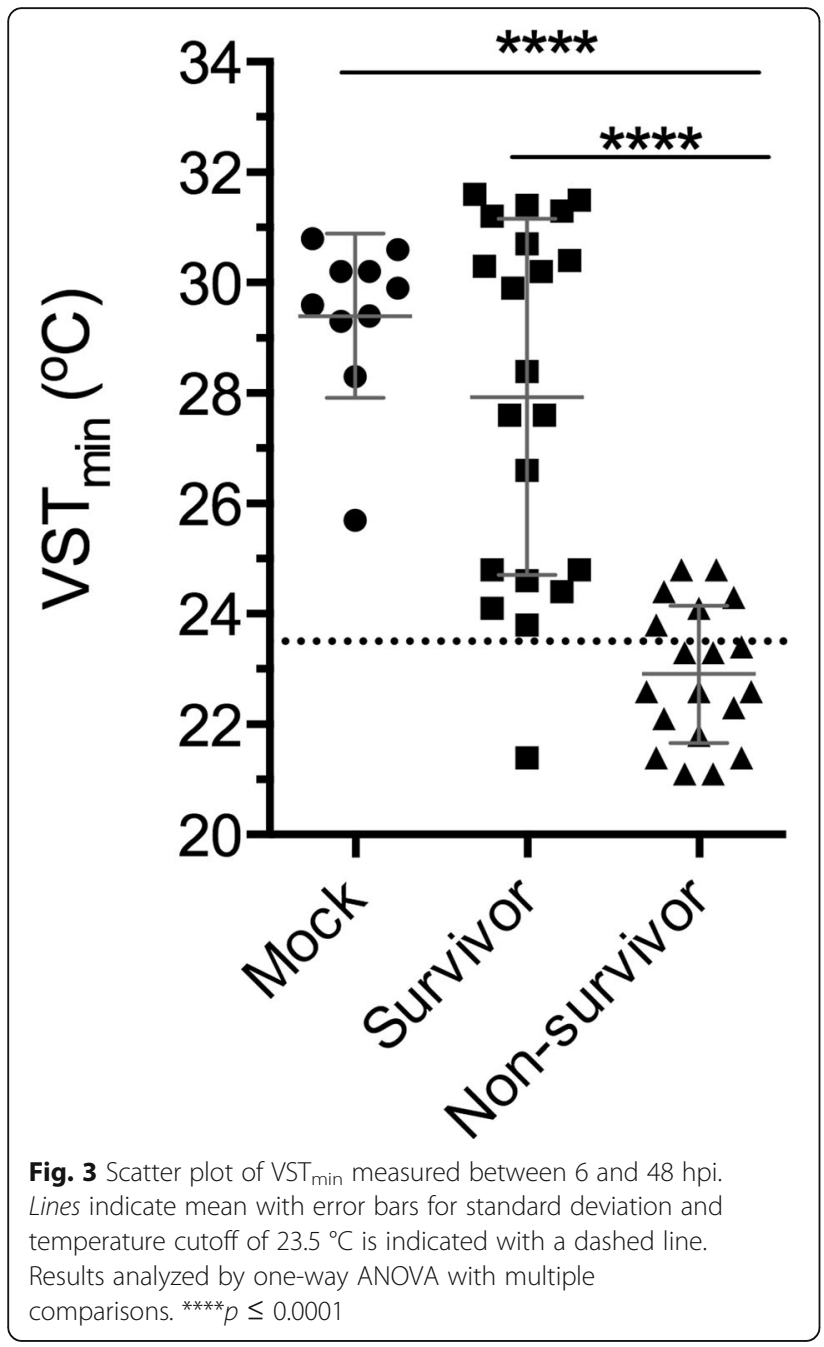

was not significantly different from mean $\mathrm{VST}_{\text {min }}$ of mock-infected mice $\left(29.4 \pm 1.5^{\circ} \mathrm{C}\right.$, Fig. 3). However, mice that later succumbed to infection exhibited significantly reduced $\mathrm{VST}_{\min }$ compared to either survivors or mocktreated animals $\left(22.9 \pm 1.2{ }^{\circ} \mathrm{C}\right.$ Fig. 3). Detailed analysis suggests that $23.5^{\circ} \mathrm{C}$ delineates between lethally and nonlethally infected mice.

Only 1 of 21 survivors exhibited VST $\leq 23.5{ }^{\circ} \mathrm{C}$ (false positive, Fig. 2b). In diagnostic terms, a test's specificity is its ability to correctly identify individuals that are not affected by a condition. In other words, specificity measures the test's true negative rate as a ratio of true negatives calls to total negative outcomes. In this case, where survival is the experiment negative outcome, 21 mice survived infection and 20 of those mice had $\mathrm{VST}_{\text {min }}>23.5$. Thus, the endpoint of VST $\leq 23.5^{\circ} \mathrm{C}$ exhibits specificity of $95 \%$. This high specificity result is important because it indicates only $5 \%$ of VST-indicated euthanizations will result in a mouse being counted as a non-survivor that otherwise would have survived infection.
Conversely, 13 of 19 non-survivors reached VST $\leq 23.5^{\circ} \mathrm{C}$ (true positive, Fig. 2c). The sensitivity of a diagnostic test indicates its ability to correctly identify those individuals affected by a condition in question. Sensitivity measures true positive rate by taking a ratio of true positive calls to total positive outcomes. In the case of VST endpoints applied to $V$. vulnificus infections, where death is the test's positive outcome, 13 non-survivor mice were predicted by their $\mathrm{VST}_{\min }$ to have lethal infection, while a total of 19 mice were ultimately non-survivors. Therefore, VST $\leq 23.5{ }^{\circ} \mathrm{C}$ has a sensitivity of $68 \%$ in predicting $V$. vulnificus infection outcome. This result means that $68 \%$ of mice that spontaneously died were indicated by VST $\leq 23.5{ }^{\circ} \mathrm{C}$. Moreover, because the remaining mice with lethal infections still proceed to death, the sensitivity of the overall experiment is actually unchanged; eventually all non-survivor outcomes are captured within the experimental context. Thus, with essentially no loss of experimental efficacy, nearly $70 \%$ of mice could have been humanely euthanized for a reduction of suffering prior to the onset of death.

Two additional measures are used to assess the diagnostic efficacy of VST $\leq 23.5^{\circ} \mathrm{C}$. Positive predictive value (PPV) is the proportion of positive diagnostic calls that are, indeed, true positives. In this case, 14 mice reached VST $\leq 23.5^{\circ} \mathrm{C} .13$ of these 14 mice were non-survivors. Therefore, the hypothermia endpoint has a PPV of $93 \%$. Conversely, the negative predictive value (NPV) is the proportion of negative test calls that are true negatives outcomes. Twenty of the 26 mice that had temperatures above the cutoff were survivors, while 6 were non-survivors. Therefore, the hypothermia endpoint VST $\leq 23.5^{\circ} \mathrm{C}$ has a NPV of $77 \%$. This analysis supports the conclusion that reduction in VST is predictive of subsequent death.

\section{VST $\leq 23.5^{\circ} \mathrm{C}$ predicts live/dead survival outcomes and reduces infection hours}

The survival data were next analyzed comparing temperature-predicted outcomes to actual outcomes. To assess the efficacy of hypothermia as an experimental endpoint when comparing different infecting bacterial strains, time-to-death and time-to-VST $\leq 23.5{ }^{\circ} \mathrm{C}$ were compared for each infecting strain in this study (Fig. 4). In the retrospective analysis (dotted lines), animals were plotted as non-survivors if either (1) the mouse exhibited VST $\leq 23.5^{\circ} \mathrm{C}$ or (2) if the mouse died even if it never reached $\leq 23.5^{\circ} \mathrm{C}$.

Statistical variation of binary outcomes (survival vs. death) was analyzed by chi-square test $\left(x^{2}\right.$, Table 2$)$. The $X^{2}$ analysis revealed no significant differences between binary survival outcomes when the hypothermia plus actual death endpoint was applied (Fig. 4, Table 2). In fact, in most cases the survival outcomes were identical 

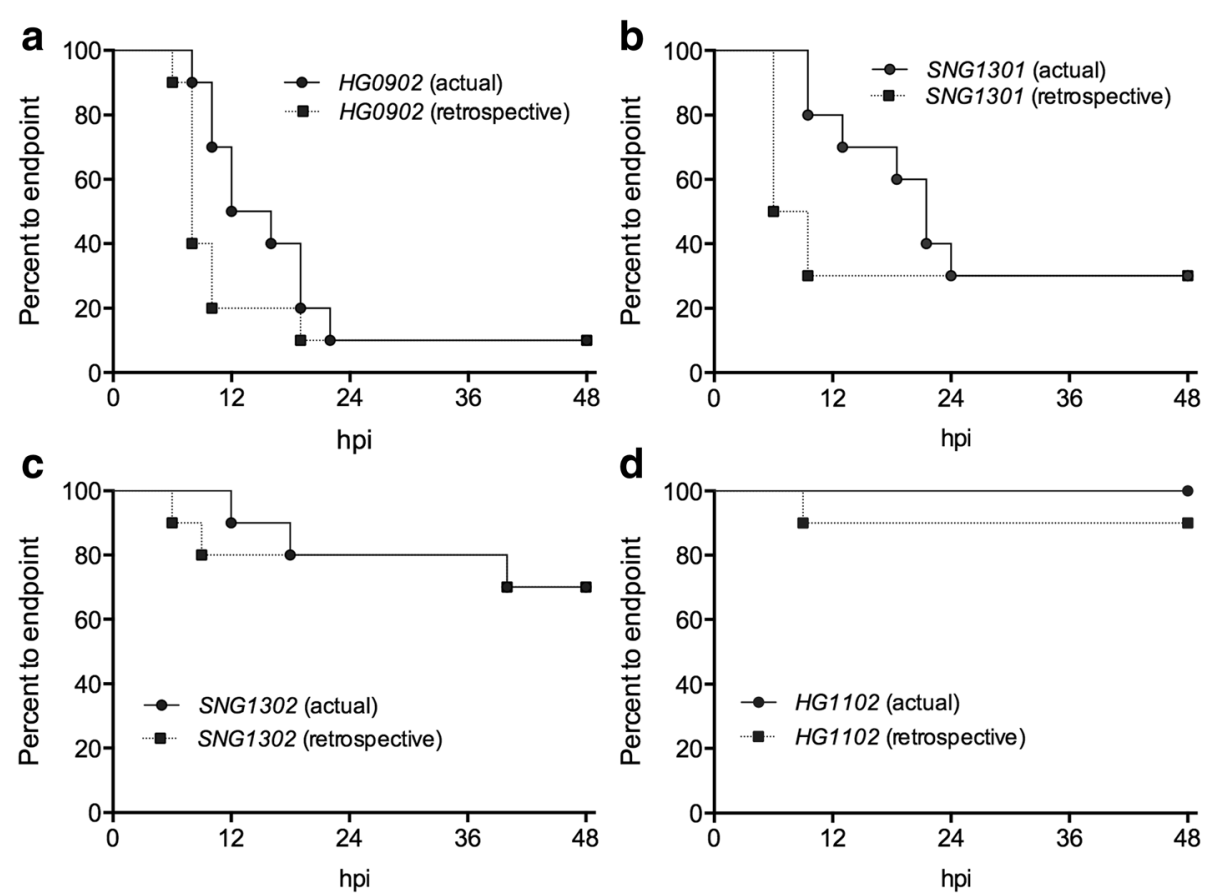

Fig. 4 Comparison of survival time for each of the V. vulnificus strains: (a) HG0902; (b) SNG1301; (c) SNG1302; (d) HG1102. Actual curves (circles connected by solid line) use death as endpoint; retrospective curves (squares connected by dotted line) use VST $\leq 23.5^{\circ} \mathrm{C}$, or death if an animal died without reaching VST $\leq 23.5^{\circ} \mathrm{C}$. Statistical analyses are summarized in Table 2

(Fig. 4a-c), but even in the case of a numerically different outcome the results were not significantly different (Fig. 4, Table 2).

VST $\leq 23.5^{\circ} \mathrm{C}$ in some cases correlated with imminent death, although many animals lived for hours prior to succumbing to infection (Fig. 2c). In non-survivors, TTD after VST $\leq 23.5^{\circ} \mathrm{C}$ had a range of $15 \mathrm{~h}$, mean of $10 \mathrm{~h}$, and S.D. of $5 \mathrm{~h}$. Therefore, while this endpoint is capable of predicting a lethal outcome, there is notable variability in the TTD. To illustrate this point, the endpoint curves (Fig. 4) were compared using the log-rank test with $95 \%$ confidence, $p<0.05$. Interestingly, there were no statistical differences between endpoint curves when analyzed by log-rank test (Table 2). Nonetheless, detailed information on the kinetics of death were lost in the hypothermia-derived curves due to time discrepancies between VST $\leq 23.5{ }^{\circ} \mathrm{C}$ and actual death (Fig. 4). While this renders the VST endpoint unsuitable for survival curve comparison, the differences in area under the survival curves (Fig. 4) illustrates the power of the alternative VST endpoint to perform its intended function: predict non-survivor outcomes, such that animals involved in the experiments can be euthanized prior to the onset of death. In the experiments illustrated in Fig. 4 , the hypothermia endpoint VST $\leq 23.5^{\circ} \mathrm{C}$ would have eliminated 135 unnecessary infection hours for mice that would eventually succumb to infection (dotted vs solid lines).

\section{Discussion}

Measurement of virulence in animal models is central to studies of bacterial pathogenesis. In lieu of death, the 'moribund condition' - generally characterized by markers such as piloerection, hunching, and respiratory irregularity - is often utilized in animal protocols as an experimental endpoint, considered more humane than death. However, moribund status can be biased due to subjectivity and inconsistency of observers, and inaccurate

Table 2 Statistical analyses of survival outcomes and curves

\begin{tabular}{lllll}
\hline Strain & \multicolumn{2}{l}{ Binary $X^{2}$ Analysis (actual vs. retro) } & & \multicolumn{2}{l}{ Log-rank curve comparison (actual vs. retro) } \\
\cline { 2 - 3 } & $x^{2}$ & $p$ & 1.8 & 0.18 \\
\hline HG0902 & 0.0 & 1.0 & 0.82 & 0.36 \\
SNG1301 & 0.0 & 1.0 & 0.0085 & 0.92 \\
SNG1302 & 0.0 & 1.0 & 1.0 & 0.32 \\
HG1102 & 1.05 & 0.30 &
\end{tabular}


if there is disconnect between observed characteristics and imminent death [21,31]. In our research group, we have noted anecdotally that there is a lack of correlation between moribund status and imminent death during $V$. vulnificus experiments. In the past 25 years, temperature monitoring has been successfully utilized to develop more refined endpoints for fungal, bacterial, and viral infection studies [19, 27, 32-34]. Retrospective analysis of additional infectious disease experiments found hypothermia to be "the most valuable characteristic for distinguishing mice that survive or succumb to infection" [17].

Core body temperature can be measured by rectal thermometry $[19,20]$, however, this method is time consuming for research staff and is thought to increase animal distress due to extensive handling [21, 26]. Moreover, intestinal probing has the potential to adversely affect experimental outcomes of a gastrointestinal pathogen due to the risk of lesion introduction [35]. The alternative method of microchip implants for thermal telemetry facilitates rapid, contact-free measurement by researchers, but requires front-end time and monetary output that is not justifiable for the acute time scale of $V$. vulnificus infections $[18,21,36]$. In addition, lesions generated at the microchip injection site are susceptible to unintentional infection [26]. Since $V$. vulnificus infects open wounds [3], the risk of subcutaneous infection is a confounding factor that renders microchip thermometry untenable for $V$. vulnificus studies.

With the advent of new technology, body surface temperature can now be measured using an external infrared thermometer [26, 27, 34]. Compared to other methods, use of a surface probe reduces animal stress, experimental complication, and initial cost [37]. Importantly, measured core and surface temperatures are correlated [37]. Correlations are particularly strong when core body temperatures are below normal, as is the case during infection-induced hypothermia [17, 37]. In the current study, infrared surface thermometry demonstrated utility in predicting disease severity during $V$. vulnificus infection and was utilized to identify a hypothermic temperature endpoint for these studies.

This study was conducted using four strains of $V$. vulnificus that differ in their known or predicted virulence. Predicted or realized differences in virulence were derived from modification of the virulence associated gene $r t x A 1$. Indeed, when the central portion of the toxin - collectively termed the effector domain region was deleted and replaced by a heterologous betalactamase coding sequence, $V$. vulnificus was significantly less virulent (Fig. 1c, HG0902 vs HG1102, [15]). To examine the necessity of a single effector domain in virulence we tested a strain SNG1301 that harbors an in-frame deletion of the $m c f$ effector domain (Fig. 1a) [23] and another that carries only the $m c f$ effector domain region.
These experiments support the conclusion that the $m c f$ coding region is neither necessary nor sufficient to change the virulence potential of $V$. vulnificus. These data provided a virulence data set as a baseline for assessing the use of VST as an endpoint for $V$. vulnificus intestinal infections.

In many cases, differences in pathogen virulence are best defined by median lethal dose $\left(\mathrm{LD}_{50}\right)$ determination. These studies need not take experimental kinetics into account, because $\mathrm{LD}_{50}$ is determined by the number of survivors and non-survivors at a given pathogen dose. In the current study, $68 \%$ of mice that spontaneously died could have been euthanized on average $10 \mathrm{~h}$ prior to death using the defined VST endpoint. At 95\% sensitivity, application of this endpoint did not compromise experimental efficacy. Thus, applying an endpoint of VST $\leq 23.5{ }^{\circ} \mathrm{C}$ during $V$. vulnificus infection has potential to considerably reduce animal suffering in studies where binary data is desired. Our finding that binary live vs. dead outcomes are predictable by hypothermia suggests that the VST alternative endpoint is appropriate for $\mathrm{LD}_{50}$ determination and should be applied to $\mathrm{LD}_{50}$ studies to reduce suffering of animals in these experiments.

$\mathrm{LD}_{50}$ measurements require large numbers of mice to test an appropriate range of doses at a level that allows for statistical power. As such, many research groups use an alternative method for determining relative virulence: infecting different groups of mice with different strains at a set dose (as in Fig. 1c) and reporting differences in the survival curves. Unlike binary $\mathrm{LD}_{50}$ studies, these curves account for time-to-death, which allows for greater statistical power with lower numbers of mice. Yet, survival curves are also considered less sensitive in their ability to detect virulence differences among strains. This study reveals that the observed lag between the VST endpoint and death renders application of a VST endpoint a poor choice for these types of studies (Fig. 4, actual vs. retrospective) due to loss of death kinetics. The curve shift actually emphasizes the utility of VST $\leq 23.5{ }^{\circ} \mathrm{C}$ in predicting lethal outcomes well before the onset of death to the experimental animals. Yet, this result likewise indicates that a VST-based endpoint does not have the same costfree benefit in survival curve experiments that it offers to $\mathrm{LD}_{50}$.

Interestingly, these results leave researchers to determine whether it is more ethical to: (A) use larger numbers of mice and perform $\mathrm{LD}_{50}$ studies, with the ability to apply a humane experimental endpoint; or (B) use smaller numbers of mice in a survival curve analysis without the option of a humane endpoint, a decision that will depend largely on the specific experimental goals of the research group in question. 


\section{Conclusions}

While hypothermia has been demonstrated as a key marker of death due to infectious disease, its relationship to survival of $V$. vulnificus infections had not previously been examined. It was here determined that surviving and non-surviving $V$. vulnificus infected mice demonstrate distinct temperature responses. Non-surviving infected mice have significantly lower $\mathrm{VST}_{\text {min }}$ as compared to surviving mice. From these data, a temperature endpoint of $23.5^{\circ} \mathrm{C}$ was empirically determined and retrospectively applied to the survival results. When used as an experimental endpoint, VST $\leq 23.5{ }^{\circ} \mathrm{C}$ exhibited sensitivity of $68 \%$ and specificity of $95 \%$. The temperature cutoff of $23.5{ }^{\circ} \mathrm{C}$ demonstrates 93\% PPV and 77\% NPV. Given the outcomes of this study, a hypothermia-based humane endpoint can be applied to $\mathrm{LD}_{50}$ studies without concern of reducing the efficacy of the experiment; binary (live vs. dead) survival outcomes are almost always numerically identical and in all cases statistically indistinguishable from survival data using death as an endpoint (Fig. 4, Table 2). As such, many hours of undue suffering would be eliminated from infection experiments.

By its very nature, an alternative endpoint aims to predict death early such that animal research subjects can be euthanized prior to death. VST $\leq 23.5{ }^{\circ} \mathrm{C}$ predicts non-survival outcomes an average of $10 \mathrm{~h}$ in advance of the event. This allows for a considerable reduction in unnecessary mouse infection hours. Nonetheless, given that a hypothermia endpoint alters experimental kinetics, these kinetic changes suggest that VST endpoint should not be applied to survival curve experiments.

\section{Abbreviations}

h: Hour(s); hpi: Hours post infection; LD 50 : Median lethal dose; n.s.: Not significantly different; NPV: Negative predictive value; PPV: Positive predictive value; S.D.: Standard deviation; TTD: Time to death; VST: Ventral surface temperature

\section{Acknowledgements}

Not applicable.

\section{Funding}

HEG was supported by a Ruth L. Kirschstein National Research Service Award from the National Institute of General Medical Sciences (5T32GM008061). KJS is the recipient of grants from the National Institute of Allergy and Infectious Diseases (R01Al092825 and R01Al098369). The funding institutions had no role in study design, data collection or manuscript preparation.

\section{Availability of data and materials}

The datasets used and/or analysed during the current study are available from the corresponding author on reasonable request.

\section{Authors' contributions}

HEG and KJFS jointly designed experiments and analyzed results. HEG performed experiments and prepared the initial manuscript. KJFS edited the manuscript. Both authors read and approved the final manuscript.

\section{Competing interests}

The authors declare that they have no competing interests.
Consent for publication

Not applicable.

\section{Ethics approval and consent to participate}

This study was carried out in strict accordance with the recommendations in the United States Public Health Service (USPHS) regulations and applicable federal and local laws regarding animal use. The protocol (Protocol No. IS00000905) was approved by the Northwestern University Institutional Animal Care and Use Committee (IACUC) and followed as detailed in methods. All efforts were made to minimize suffering.

\section{Publisher's Note}

Springer Nature remains neutral with regard to jurisdictional claims in published maps and institutional affiliations.

Received: 10 February 2017 Accepted: 9 June 2017

Published online: 19 June 2017

\section{References}

1. Oliver JD. The Biology of Vibrio vulnificus. Microbiol Spectr. 2015;3(3):VE-0012014.

2. Jones MK, Oliver JD. Vibrio vulnificus: disease and pathogenesis. Infect Immun. 2009;77(5):1723-33.

3. Horseman MA, Surani S. A comprehensive review of Vibrio vulnificus: an important cause of severe sepsis and skin and soft-tissue infection. Int J Infect Dis. 2011;15(3):e157-66.

4. Froelich BA, Noble RT. Vibrio bacteria in raw oysters: managing risks to human health. Philos Trans R Soc Lond B Biol Sci. 2016;371(1689):20150209.

5. Jones B, Burkhart J, Sonpavde G. Danger on a half shell: Vibrio vulnificus septicemia. Am J Med. 2015;128(5):475-6.

6. Hoffman S, Maculloch B, Batz M. Economic burden of major foodborne illnesses acquired in the United States, EIB-140. United States Department of Agriculture, Economic Research Service. 2015. https://www.ers.usda.gov/ webdocs/publications/43984/52807_eib140.pdf?

7. Chao CH, Duh RW, Liu CY, Lou JP, Chen CK. Experience of six patients with Vibrio vulnificus septicemia. Zhonghua Yi Xue Za Zhi (Taipei). 1992;49(5):335-42.

8. Menon MP, Yu PA, Iwamoto M, Painter J. Pre-existing medical conditions associated with Vibrio vulnificus septicaemia. Epidemiol Infect. 2013:1-4.

9. Lee $\mathrm{SH}$, Chung BH, Lee WC. Retrospective analysis of epidemiological aspects of Vibrio vulnificus infections in Korea in 2001-2010. Jpn J Infect Dis. 2013;66(4):331-3.

10. Inoue Y, Ono T, Matsui T, Miyasaka J, Kinoshita Y, Ihn H. Epidemiological survey of Vibrio vulnificus infection in Japan between 1999 and 2003. J Dermatol. 2008:35(3):129-39.

11. Bisharat N, Agmon V, Finkelstein R, Raz R, Ben-Dror G, Lerner L, et al. Clinical epidemiological, and microbiological features of Vibrio vulnificus biogroup 3 causing outbreaks of wound infection and bacteraemia in Israel. Israel Vibrio Study Group. Lancet. 1999:354(9188):1421-4

12. Jeong HG, Satchell KJ. Additive function of Vibrio vulnificus MARTX $\mathrm{V}_{V}$ and VvhA cytolysins promotes rapid growth and epithelial tissue necrosis during intestinal infection. PLoS Pathog. 2012;8(3):e1002581.

13. Kwak JS, Jeong HG, Satchell KJ. Vibrio vulnificus rtxA1 gene recombination generates toxin variants with altered potency during intestinal infection. Proc Natl Acad Sci U S A. 2011;108(4):1645-50.

14. Kashimoto T, Iwasaki C, Gojo M, Sugiyama H, Yoshioka K, Yamamoto Y, et al Vibrio vulnificus detected in the spleen leads to fatal outcome in a mouse oral infection model. FEMS Microbiol Lett. 2015;362(7):fnv0005.

15. Gavin HE, Beubier NT, Satchell KJ. The effector domain region of the Vibrio vulnificus MARTX toxin confers biphasic epithelial barrier disruption and is essential for systemic spread from the intestine. PLoS Pathog. 2017;13(1): e1006119.

16. Olfert ED, Godson Dale L. Humane endpoints for infectious disease animal models. Inst Lab Anim Res J. 2000:41(2):99-104.

17. Trammell RA, Toth LA. Markers for predicting death as an outcome for mice used in infectious disease research. Comp Med. 2011;61(6):492-8.

18. Vlach KD, Boles JW, Stiles BG. Telemetric evaluation of body temperature and physical activity as predictors of mortality in a murine model of staphylococcal enterotoxic shock. Comp Med. 2000:50(2):160-6.

19. Wong JP, Saravolac EG, Clement JG, Nagata LP. Development of a murine hypothermia model for study of respiratory tract influenza virus infection. Lab Anim Sci. 1997;47(2):143-7. 
20. Soothill JS, Morton DB, Ahmad A. The HID50 (hypothermia-inducing dose 50): an alternative to the LD50 for measurement of bacterial virulence. Int J Exp Pathol. 1992;73(1):95-8.

21. Toth LA. Defining the moribund condition as an experimental endpoint for animal research. ILAR J. 2000;41(2):72-9.

22. Kim BS, Gavin HE, Satchell KJ. Distinct roles of the repeat-containing regions and effector domains of the Vibrio vulnificus multifunctional-autoprocessing repeats-in-toxin (MARTX) toxin. MBio. 2015;6(2):e00324-15.

23. Agarwal S, Zhu Y, Gius DR, Satchell KJ. The Makes Caterpillars Floppy (MCF)like domain of Vibrio vulnificus induces mitochondrion-mediated apoptosis. Infect Immun. 2015:83(11):4392-403.

24. Fullner KJ, Mekalanos JJ. Genetic characterization of a new type IV-A pilus gene cluster found in both classical and El Tor biotypes of Vibrio cholerae. Infect Immun. 1999;67(3):1393-404.

25. Olivier V, Queen J, Satchell KJ. Successful small intestine colonization of adult mice by Vibrio cholerae requires ketamine anesthesia and accessory toxins. PLoS One. 2009:4(10):e7352.

26. Warn PA, Brampton MW, Sharp A, Morrissey G, Steel N, Denning DW, et al. Infrared body temperature measurement of mice as an early predictor of death in experimental fungal infections. Lab Anim. 2003;37(2):126-31.

27. Hankenson FC, Ruskoski N, van Saun M, Ying GS, Oh J, Fraser NW. Weight loss and reduced body temperature determine humane endpoints in a mouse model of ocular herpesvirus infection. J Am Assoc Lab Anim Sci. 2013:52(3):277-85.

28. Clement JG, Mills P, Brockway B. Use of telemetry to record body temperature and activity in mice. J Pharmacol Methods. 1989:21(2):129-40.

29. Krarup A, Chattopadhyay P, Bhattacharjee AK, Burge JR, Ruble GR. Evaluation of surrogate markers of impending death in the galactosamine-sensitized murine model of bacterial endotoxemia. Lab Anim Sci. 1999;49(5):545-50.

30. Gargiulo S, Greco A, Gramanzini M, Esposito S, Affuso A, Brunetti A, et al, Mice anesthesia, analgesia, and care, part 1: anexthetic considerations in preclinical research. ILAR J. 2012;53(1):E55-69.

31. Toth LA. The moribund state as an experimental endpoint. Contemp Top Lab Anim Sci. 1997;36(3):44-8.

32. Toth LA, Rehg JE, Webster RG. Strain differences in sleep and other pathophysiological sequelae of influenza virus infection in naive and immunized mice. J Neuroimmunol. 1995:58(1):89-99.

33. Stiles BG, Campbell YG, Castle RM, Grove SA. Correlation of temperature and toxicity in murine studies of staphylococcal enterotoxins and toxic shock syndrome toxin 1. Infect Immun. 1999;67(3):1521-5.

34. Bast DJ, Yue M, Chen X, Bell D, Dresser L, Saskin R, et al. Novel murine model of pneumococcal pneumonia: use of temperature as a measure of disease severity to compare the efficacies of moxifloxacin and levofloxacin. Antimicrob Agents Chemother. 2004;48(9):3343-8.

35. Clement JG. Experimentally induced mortality following repeated measurement of rectal temperature in mice. Lab Anim Sci. 1993:43(4):381-2.

36. Kort WJ, Hekking-Weijma JM, TenKate MT, Sorm V, VanStrik R. A microchip implant system as a method to determine body temperature of terminally ill rats and mice. Lab Anim. 1998;32(3):260-9.

37. Newsom DM, Bolgos GL, Colby L, Nemzek JA. Comparison of body surface temperature measurement and conventional methods for measuring temperature in the mouse. Contemp Top Lab Anim Sci. 2004;43(5):13-8.

\section{Submit your next manuscript to BioMed Central and we will help you at every step:}

- We accept pre-submission inquiries

- Our selector tool helps you to find the most relevant journal

- We provide round the clock customer support

- Convenient online submission

- Thorough peer review

- Inclusion in PubMed and all major indexing services

- Maximum visibility for your research

Submit your manuscript at www.biomedcentral.com/submit

CBiomed Central 